\title{
gbpA and chiA genes are not uniformly distributed amongst diverse Vibrio cholerae
}

\author{
Thea G. Fennell1,2†, Grace A. Blackwell ${ }^{1,3}$, Nicholas R. Thomson ${ }^{1,4}$ and Matthew J. Dorman ${ }^{1,2, *}$
}

\begin{abstract}
Members of the bacterial genus Vibrio utilize chitin both as a metabolic substrate and a signal to activate natural competence. Vibrio cholerae is a bacterial enteric pathogen, sub-lineages of which can cause pandemic cholera. However, the chitin metabolic pathway in $V$. cholerae has been dissected using only a limited number of laboratory strains of this species. Here, we survey the complement of key chitin metabolism genes amongst 195 diverse $V$. cholerae. We show that the gene encoding GbpA, known to be an important colonization and virulence factor in pandemic isolates, is not ubiquitous amongst $\mathrm{V}$. cholerae. We also identify a putatively novel chitinase, and present experimental evidence in support of its functionality. Our data indicate that the chitin metabolic pathway within V. cholerae is more complex than previously thought, and emphasize the importance of considering genes and functions in the context of a species in its entirety, rather than simply relying on traditional reference strains.
\end{abstract}

\section{DATA SUMMARY}

The authors confirm that all supporting data, code and protocols have been provided within the article or through supplementary data files.

(1) No whole-genome sequencing data were generated in this study. Accession numbers for the publicly available sequences used for these analyses are listed in Tables 2 and S1 (available in the online version of this article), and the Methods.

(2) Metadata, accession numbers and references for each genome included in this study are provided in Table S1, deposited in Figshare: (https://dx.doi.org/10.6084/m9. figshare.14398688).

(3) Supplementary tables, figures and references are available in the Supplementary Material file, deposited in Figshare: (https://dx.doi.org/10.6084/m9.figshare. 14398688).

(4) All other data which underpin the figures in this paper, including pangenome data matrices, modified and unmodified sequence alignments and phylogenetic trees, original images of gels and immunoblots, raw fluorescence data, amplicon sequencing reads, and the $\mathrm{R}$ code used to generate Fig. 5, are available in Figshare: https://dx.doi.org/10.6084/m9.figshare.13169189 (DOI).

\section{INTRODUCTION}

The genus Vibrio of marine gammaproteobacteria contains a number of virulent human pathogens, of significant public health concern [1]. Most notorious of these pathogens is Vibrio cholerae, members of which are the aetiological agent of cholera in humans [2,3]. Two biochemically defined and distinct $V$. cholerae biotypes are associated with cholera pandemics. Classical biotype $V$. cholerae are believed to have caused the first six pandemics [2-4], whilst the current seventh pandemic (1961 to the present) is attributed to El Tor biotype $V$. cholerae $[5,6]$. Genomic evidence has shown that classical $V$. cholerae form a discrete phylogenetic lineage from the lineage causing the seventh cholera pandemic, dubbed

Received 11 February 2021; Accepted 26 April 2021; Published 08 June 2021

Author affiliations: 'Wellcome Sanger Institute, Wellcome Genome Campus, Hinxton, CB10 1SA, UK; ${ }^{2}$ Churchill College, Storey's Way, Cambridge, CB3

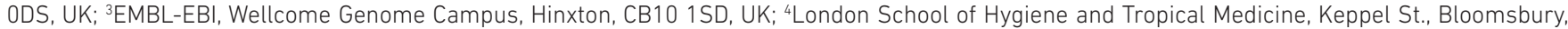
London, WC1E 7HT, UK.

*Correspondence: Matthew J. Dorman, mjd211@cam.ac.uk; md25@sanger.ac.uk

Keywords: chitin; chitinase; ChiA; cholera; GbpA; Vibrio cholerae.

Abbreviations: ABC transporter, ATP-binding cassette transporters; CmR, chloramphenicol resistant; dNTP, deoxyribonucleoside triphosphate:

GlcNAc, $N$-acetyl- $\beta$-D-glucosamine; MSHA, mannose-sensitive haemagglutinin; 4-MU, 4-methylumbelliferyl; PTS transporter, phosphotransferase transporter; SNV, single nucleotide variant; StrR, streptomycin resistant.

†Present address: Sainsbury Laboratory, University of Cambridge, Bateman Street, Cambridge, UK.

Data statement: All supporting data, code and protocols have been provided within the article or through supplementary data files. Three supplementary tables and seven supplementary figures are available with the online version of this article.

000594 @ 2021 The Authors 
the seventh pandemic El Tor lineage (7PET) [7-12]. It is to these two pandemic lineages that commonly used El Tor and classical biotype laboratory strains belong. Although cholera is estimated to cause 100000 deaths annually worldwide [13], other Vibrio species can also cause enteric and extraintestinal disease in humans. For example, Vibrio vulnificus can cause septicaemia and systemic infection in humans [14], and Vibrio parahaemolyticus can cause gastrointestinal infection, septicaemia and wound infections [1, 15]. Other vibrios may be pathogenic to livestock and other animals, such as Vibrio nigripulchritudino, which is a pathogen of farmed shrimp [16, 17], Vibrio anguillarum, which causes vibriosis in multiple species of fish [18], and Vibrio harveyi, which is an important pathogen of both fish and shrimp [19].

In spite of differences in the types of disease which Vibrio species may cause, there are several commonalities amongst members of this genus. For example, it has been suggested that the ability to grow on chitin is a ubiquitous phenotype amongst the Vibrionaceae [20], and therefore that all Vibrio species are capable of metabolizing chitin, a highly abundant polymer of $N$-acetyl- $\beta$-D-glucosamine (GlcNAc) [21]. This is directly relevant to the environmental lifestyles of vibrios - for example, V. vulnificus colonizes and grows on the surface of chitinaceous animals such as shellfish [22]. $V$. parahaemolyticus secretes a chitinase and can adsorb onto particulate chitin and copepods [23]. Similarly, V. cholerae can metabolize chitin [24], has chitinase activity and can adsorb on chitinous substrates [25], and can colonize chitinous surfaces such as those of copepods [26]. Chitin metabolism is linked to other aspects of Vibrio biology, including the regulation of natural competence [27-29], and to the survival of $V$. cholerae in the context of the intestine during an infection [30].

The pathways by which chitin is degraded and utilized by $V$. cholerae have been described in detail [31], as it has been in other members of the genus (e.g. [32-39]). Although a comprehensive review of the chitin utilization pathway is beyond the scope of this paper, it is important to appreciate the complexity of this pathway. Chitin degradation, import and metabolism in $V$. cholerae involves at least 27 proteins, 24 encoded by genes on chromosome 1 , and three by genes on the smaller chromosome 2 [20]. Here, focus will be directed to the initial stages of chitin metabolism - adhesion to a chitinous substrate, and expression of extracellular degradative chitinase enzymes.

The first step in chitin metabolism is the attachment of $V$. cholerae to chitinaceous surfaces through interactions with GlcNAc. This is mediated both by the mannosesensitive haemagglutinin (MSHA) pilus and the chitin adhesin GbpA (encoded by VC_A0811, accession no. AAF96709.1) $[24,40]$. Although GbpA was initially identified as a putative chitinase enzyme [24], it was shown to be an adhesin induced by GlcNAc which enabled $V$. cholerae to attach to chitinous substrates [24]. Subsequently, it was found that as well as mediating attachment of $V$. cholerae to chitin, GbpA is also required for the successful colonization

\section{Impact Statement}

It is thought that the ability to metabolize chitin is ubiquitous amongst Vibrio species, and that this enables these species to survive in aqueous and estuarine environmental contexts. Although chitin metabolism pathways have been detailed in several members of this genus, little is known about how these processes vary within a single Vibrio species. Here, we present the distribution of genes encoding key chitinase and chitin-binding proteins across diverse Vibrio cholerae, and show that our canonical understanding of this pathway in this species is challenged when isolates from non-pandemic $V$. cholerae lineages are considered alongside those linked to pandemics. Furthermore, we show that genes previously thought to be species core genes are not in fact ubiquitous, we identify novel components of the chitin metabolic cascade in this species, and we present functional validation for these observations.

of the intestine [40]. This is thought to occur through interactions with mucin - GbpA interacts with mucin in the intestine, and $g b p A$ transcription increases upon exposure of $V$. cholerae to mucin [41]. The crystal structure and domain architecture of GbpA have been determined [42], and the fourth domain of GbpA is structurally similar to the chitin-binding domain of known chitinases [42]. Evidence also suggests that GbpA has lytic polysaccharide monooxygenase activity [43], and that GbpA activity is higher at low population densities due to the activity of quorum-sensingregulated proteases [44].

Once $V$. cholerae adheres to a chitinaceous surface, extracellular endochitinase enzymes are required for the bacterium to hydrolyse complex chitin polymers into oligosaccharides which can be imported into the cell for further metabolism [35]. As many as seven putative endochitinases have been identified in $V$. cholerae $[20,45,46]$, two of which, ChiA-1 (encoded by VC_1952, accession no. AAF95100.1) and ChiA-2 (encoded by VC_A0027, accession no. AAF95941.1), are the principal chitinases required for $V$. cholerae chitin catabolism $[24,31,45,47]$. ChiA-1 was first shown to be an extracellular chitinase in 1998 [48]; subsequently, ChiA-2 was shown to be important for intestinal colonization and for metabolizing mucin in the intestine by V. cholerae strain N16961 (N16961) [30]. ChiA-2 is also the most highly expressed chitinase in El Tor biotype V. cholerae strain E7946 [45]. Both ChiA-1 and ChiA-2 are essential for $V$. cholerae to grow in media supplemented with colloidal chitin [24]. Once chitin oligomers have been digested by extracellular chitinases, the resultant oligosaccharides are thought to enter the bacterial periplasm via the chitoporin ChiP (encoded by VC_0972, accession no. AAF94134.1) and by other as-yet-uncharacterized porins $[24,36,49]$, and subsequently transported to the cytoplasm 


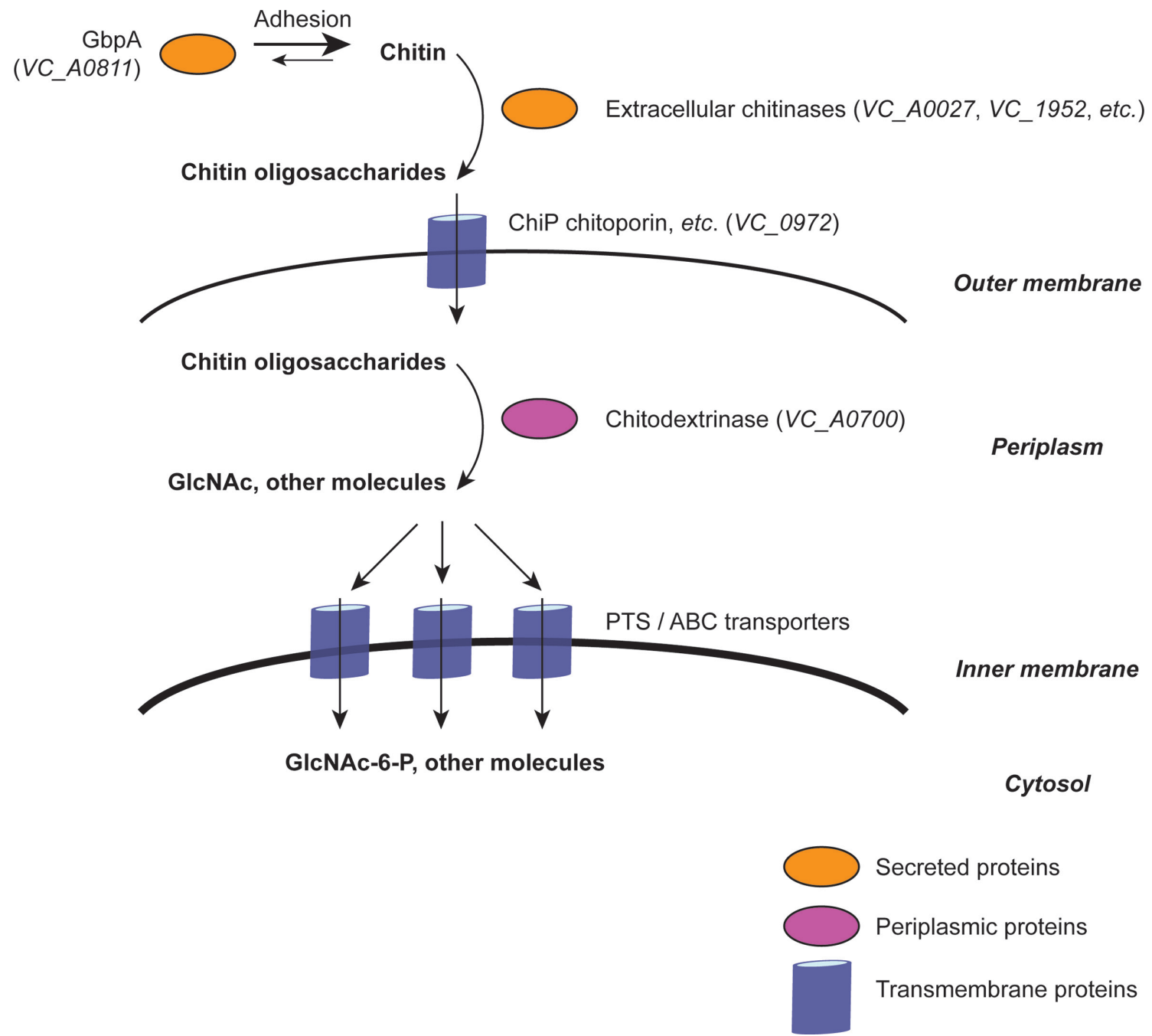

Fig. 1. Initial steps in V. cholerae chitin uptake and catabolism. Schematic summarizing the principal stages in chitin degradation and import by V. cholerae. Comprehensive descriptions of this pathway are reported in the literature $[20,36]$. The MSHA adhesin has not been included in this diagram.

via phosphotransferase (PTS) and ABC-type transporters [20,36] (Fig. 1).

Previous work used the genomes of 20 diverse Vibrionaceae (including seven $V$. cholerae) to determine the presence and absence of genes involved in metabolizing chitin across this family of bacteria [20]. However, it is important to note that the chitin degradation pathway of $V$. cholerae has been described using reference strains of the species (particularly N16961 [24]), and although data exist on how the chitin catabolism pathway varies amongst members of the genus Vibrio [20], less is known about how this pathway varies within a single species. This is particularly relevant because emerging evidence suggests that non-7PET lineages of $V$. cholerae cause different patterns of disease, even if they harbour some or all of the canonical pathogenicity determinants associated with cholera cases [8]. However, because the chitin metabolic pathway has principally been studied in N16961, a 7PET strain, we know little about the extent to which it varies amongst non-pandemic members of $V$. cholerae.

In this study, we focused specifically on genes that encode components of the initial steps of the chitin degradation pathway across $V$. cholerae. We focused on these because the functions of many of these genes have been characterized experimentally, and we sought to determine how well the observations in the literature reflect the true distribution of these genes, and their functions, across a diverse species. We generated a pangenome from 195 annotated $V$. cholerae genome sequences, which were chosen to obtain as balanced and unbiased a view of $V$. cholerae as possible (i.e. without focusing solely on epidemic and pandemic lineages). We find that the distribution of these genes is not uniform within $V$. cholerae, and we identify variation amongst the chitinases encoded by diverse $V$. cholerae. 
Table 1. Strains, plasmids and oligonucleotides used in this study

Restriction enzyme recognition sites are underlined. The primer sequence incorporating a C-terminal 6 xHis translational fusion into chi $A-3$ is presented in lowercase, and the sequences of ribosome binding sites, start and STOP codons are in bold. CmR, chloramphenicol resistant; StrR, streptomycin resistant.

\begin{tabular}{|c|c|c|c|}
\hline Internal strain ID & Strain name & Genotype/details & Source/reference \\
\hline MJD382 & NCTC 30 & Non-pandemic $V$. cholerae harbouring chiA-3 & $\begin{array}{l}\text { Thomson lab stocks; National } \\
\text { Centre for Type Cultures, batch 3, } \\
\text { sequenced previously [65] }\end{array}$ \\
\hline \multicolumn{4}{|l|}{ Escherichia coli } \\
\hline MJD1507 & NEB NiCo21(DE3) & 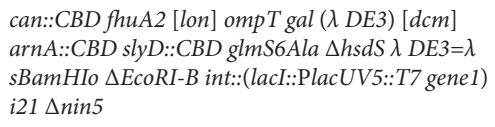 & New England Biolabs \\
\hline MJD1499 & MJD1499 & NiCo21(DE3) harbouring pBAD33. CmR & This study \\
\hline Plasmid name & \multicolumn{2}{|l|}{ Genotype/details } & Source/reference \\
\hline pBAD33 & \multicolumn{2}{|c|}{ Arabinose-inducible expression plasmid; pACYC184 replication origin. $\mathrm{CmR}$} & Thomson lab stocks [88] \\
\hline pMJD157 & \multicolumn{2}{|l|}{ chiA-3 cloned into pBAD33. CmR } & This study \\
\hline Primer ID & Other name & \multicolumn{2}{|l|}{ Sequence $5^{\prime}-3^{\prime}$} \\
\hline oMJD202 & TF_SacI_Chitinase_F & \multicolumn{2}{|c|}{ GCGAGCTCAGGAGGATCTCTATGAAAAAAACAGTCATTGCTACC } \\
\hline oMJD203 & TF_Chitinase_6xHis_STOP_SalI_R & \multicolumn{2}{|c|}{ GCGTCGACTTAgtgatggtgatggtgatgTTTGATCGTTTCAAACATGGT } \\
\hline
\end{tabular}

We also identify a putatively novel chitinase gene, and present experimental evidence in support of its functional classification.

\section{METHODS}

\section{Strains, plasmids and oligonucleotides}

Strains, plasmids and oligonucleotide primers (SigmaAldrich) used for experimental work in this study are listed in Table 1. Bacteria were cultured routinely on LB media supplemented with chloramphenicol $\left(10 \mu \mathrm{g} \mathrm{ml}^{-1}\right.$; LB-Cm) where appropriate.

\section{Genome sequences and accession numbers}

The 198 genome sequences used to calculate the pangenome described in this paper are listed in Table S1. Accession numbers for additional chromosome sequences to which the text refers are as follows: $V$. harveyi chromosome 2 (accession no. CP009468.1); V. parahaemolyticus chromosome 2 (accession no. BA000032.2). Accession numbers for the chitinase protein sequences referred to in Hunt et al. [20] and used for BLASTp comparisons are listed in Table 2.

\section{Genome assemblies}

$V$. cholerae genome sequences were assembled from short-read data using SPAdes v3.8.2 [50], as part of a high-throughput pipeline [51]. Assemblies were annotated automatically using Prokka v1.5 [52] and a genus-specific reference database [53]. If raw sequencing reads were unavailable for genome sequences, assemblies were downloaded and similarly annotated using the automated Prokka-based pipeline.

\section{Pangenome and phylogenetic calculations}

A pangenome was produced from 198 Prokka-annotated genome assemblies using Roary v3.12.0 [54] (parameters: '-p 10 -e --mafft -s -cd 97'). A core-gene alignment of 2520 genes and 1096140 nucleotides was produced from this pangenome calculation. The alignment was trimmed using trimAl v1.4.1 [55] and used to produce an alignment of 183896 single 
Table 2. Pairwise BLASTp alignments between chiA-3 and chitinases from Hunt et al. [20]

No significant alignment was found between chiA-3 and VAS14_08875, VAS14_08910,V12G01_01435, V12G01_22308 or VF1146. A multiple sequence alignment containing each of these protein sequences has been included in the Figshare repository for this study.

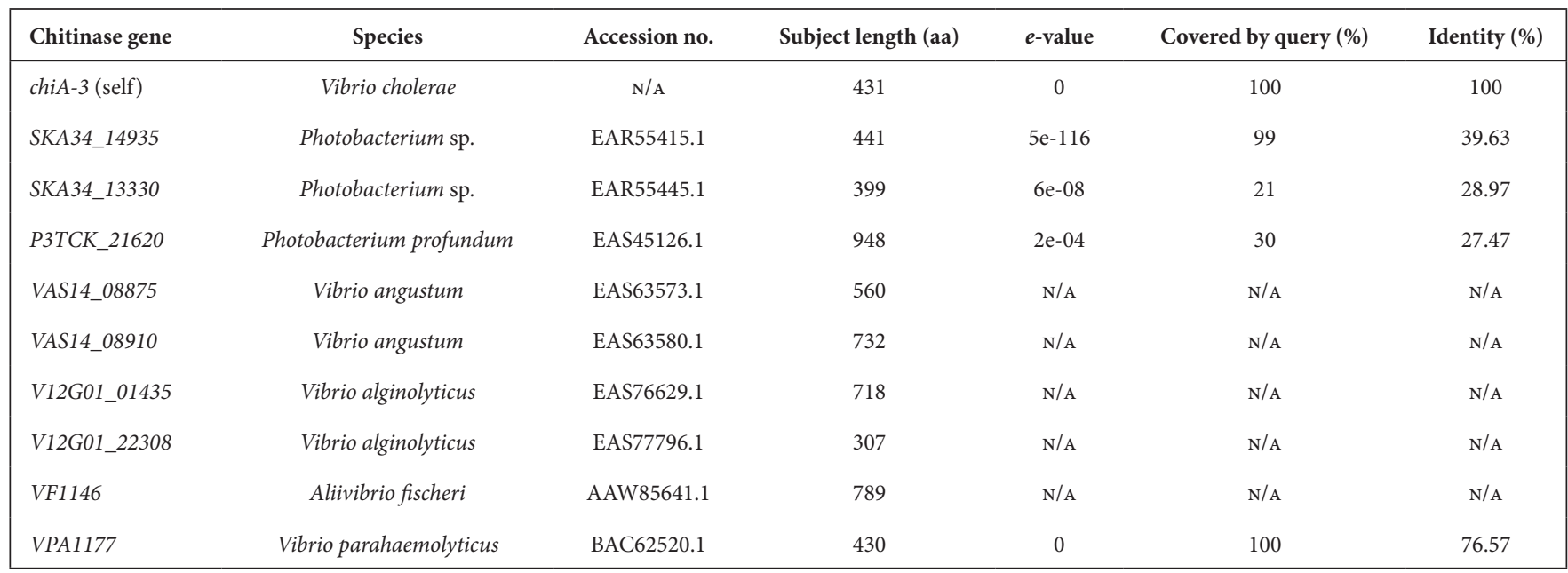

nucleotide variants (SNVs) using SNP-sites v2.5.1 [56]. A maximum-likelihood phylogeny was produced using IQ-Tree v1.6.10 [57] from the SNV-only alignment (options: '-nt 10 -m GTR+ASC -bb 5000 -alrt 5000').

\section{Protein sequence alignments, domain prediction and comparative genomics}

Protein sequences were aligned using BLASTp [58] and were annotated using the InterProScan web server [59]. Comparative genomic figures were generated using BLASTn [58] sequence alignments and visualized using ACT v13 and v18.0.2 [60], and Easyfig v2.2.2 [61].

\section{Confirmation of gene presence/absence by mapping}

Reads were mapped to reference sequences using SMALT v0.7.4 (https://www.sanger.ac.uk/tool/smalt-0/) and the method described by Harris et al. [62], as part of automated analysis pipelines run by Wellcome Sanger Institute Pathogen Informatics. All of the software developed by Pathogen Informatics is freely available for download from GitHub under an open source licence, GNU GPL 3 (https://github. com/sanger-pathogens/vr-codebase). Ordered BAM files were visualized against reference sequences using Artemis v16 and v18.0.2, which incorporate BamView $[63,64]$.

\section{Molecular cloning}

Plasmid DNA was extracted from Escherichia coli using the QIAprep Spin Miniprep kit (Qiagen; \#27104). Genomic DNA (gDNA) was extracted from NCTC 30 as described previously [65]. Cloning intermediates were purified using the QIAquick PCR Purification kit (Qiagen; \#28104). gDNA from NCTC 30 was used as a template from which to amplify chiA-3 using primers oMJD202 and oMJD203, high-fidelity Phusion Hot Start Flex polymerase [New England Biolabs (NEB); \#M0535S] using the supplied GC buffer, DMSO (3\%,v/v, final concentration) and dNTPs (Thermo Scientific; \#R0191). Twenty-nine PCR cycles were performed using the manufacturer's protocol (annealing temperature: $55^{\circ} \mathrm{C}$, extension time: $2 \mathrm{~min}$ ). The amplicon was purified and digested using 30 units of SacI-HF and SalI-HF (NEB; \#R3156S and R3138S respectively) at $37^{\circ} \mathrm{C}$ for $45 \mathrm{~min}$. pBAD33 was similarly treated with SacI-HF and SalI-HF, and after $15 \mathrm{~min}$ incubation at $37^{\circ} \mathrm{C}$, the plasmid digestion was supplemented with 1.5 units of recombinant shrimp alkaline phosphatase (rSAP; NEB; \#M0371S). Digested insert and vector were purified and ligated together at room temperature for $30 \mathrm{~min}$ using T4 DNA ligase (NEB; \#M0202S) in approximately a 3:1 molar ratio. Chemically competent 10-beta E. coli (NEB; \#C3019I) were transformed with ligated DNA according to the manufacturer's instructions, and transformants were selected for on LB agar supplemented with chloramphenicol $\left(10 \mu \mathrm{g} \mathrm{ml}^{-1}\right)$.

Chloramphenicol-resistant colonies were resuspended in $30 \mu \mathrm{l}$ PBS. A screen for clones containing an insert into pBAD33 was carried out using $1 \mu \mathrm{l}$ of this suspension as a PCR template using primers oMJD204 and oMJD205 and OneTaq Quickload 2X Master Mix (NEB; \#M0486S), according to the manufacturer's instructions (annealing temperature $45^{\circ} \mathrm{C}$, extension time $3 \mathrm{~min}$ ). Plasmids were extracted from overnight cultures of clones from which PCR produced an amplicon of the expected size (1548 bp). The presence of an insertion into pBAD33 was verified by digesting purified plasmid DNA with SacI-HF and SalI-HF as described above. Plasmids were then sequence-confirmed by amplicon sequencing (GATC/Eurofins Genomics) in both directions across the pBAD33 multiple cloning site using primers oMJD204 and oMJD205. Sequence-verified plasmids were transformed into chemically competent NiCo21(DE3) cells (NEB; \#C2529H) following the manufacturer's instructions, and these transformants were used for protein expression purposes. 


\section{Protein expression and immunoblotting}

Single colonies of NiCo21(DE3) harbouring pMJD157 and pBAD33 (empty vector) were inoculated into $3 \mathrm{ml} \mathrm{LB}-\mathrm{Cm}$ and cultured at $37^{\circ} \mathrm{C}$ with shaking (200 r.p.m.) for $8 \mathrm{~h}$. These were then diluted 1:100 into baffled flasks containing $25 \mathrm{ml} \mathrm{LB}-\mathrm{Cm}$ supplemented with either $\mathrm{D}-(+)$-glucose $(\mathrm{BDH}$, now VWR; \#101176K) or L-(+)-arabinose (Sigma-Aldrich; \#A3256), both at $0.4 \%(\mathrm{w} / \mathrm{v})$ final concentration. These cultures were grown for $18 \mathrm{~h}$ at $23^{\circ} \mathrm{C}$ with shaking (200 r.p.m.). Cells were collected by centrifugation $(3900 \mathrm{~g}, 5 \mathrm{~min})$ and the supernatant was filter-sterilized $(0.22 \mu \mathrm{m})$ and stored at $-20^{\circ} \mathrm{C}$. Cell pellets were lysed in $3 \mathrm{ml}$ BugBuster HT (Millipore; \#70922-4) for $30 \mathrm{~min}$ at room temperature on a rotator. Debris was collected by centrifugation $(3900 \mathrm{~g}, 5 \mathrm{~min}$ ) and discarded. Lysates were stored at $-20^{\circ} \mathrm{C}$.

Sixty microlitres of filtered supernatants and lysates was mixed 1:1 with $2 \times$ tris-glycine-SDS sample buffer (Invitrogen; \#LC2676), boiled at $100^{\circ} \mathrm{C}$ for $5 \mathrm{~min}$, and $30 \mu \mathrm{l}$ of each sample was used to load duplicate NuPAGE 4-12\% Bis-Tris acrylamide gels (Invitrogen; \#NP0321) which were electrophoresed simultaneously, in the same gel tank. Stained and unstained protein ladders (NEB; \#P7719S and \#P7717S, Invitrogen; \#LC5925) were used for size estimation where appropriate. One gel of the pair was stained with InstantBlue (Expedeon/Abcam; \#ISB1L) according to the manufacturer's instructions prior to imaging; the other was used for Western immunoblotting.

For Western blotting, electrophoresed proteins were transferred from an acrylamide gel onto a nitrocellulose membrane using the iBlot 2 dry blotting system and transfer stack (ThermoFisher; \#IB21001 and \#IB23001). After transfer, the membrane was blocked for $3 \mathrm{~h}$ in $5 \%(\mathrm{w} / \mathrm{v})$ Marvel milk powder dissolved in PBS-Tween 20 (Marvel-PBS-T) at $4^{\circ} \mathrm{C}$, with rocking. An antibody recognizing the $6 \mathrm{xHis}$ epitope and directly conjugated to horseradish peroxidase (Abcam; \#ab1187) was diluted in Marvel-PBS-T according to the manufacturer's instructions and used to probe the membrane for $30 \mathrm{~min}$ at $4{ }^{\circ} \mathrm{C}$, with rocking. The membrane was then washed in PBS-T for $15 \mathrm{~min}$ three times, and then incubated with Clarity Western ECL substrate (Bio-Rad; \#170-5060) for $5 \mathrm{~min}$. Luminescence signal was allowed to decay overnight, and the blot was then imaged with Amersham Hyperfilm ECL film (GE Healthcare; \#28906836). Coloured protein size standards were marked manually on the developed film.

\section{Chitinase assay}

Chitinase activity was assayed using fluorogenic substrates (Sigma-Aldrich; \#CS1030). The kit was used according to the manufacturer's instructions, with the following modifications, Ten microlitres of cell lysate or supernatant was used per assay well. Five microlitres of the supplied chitinase control enzyme was used per positive control reaction, rather than a 1:200 dilution of the control enzyme, to ensure that fluorescence was detectable. Assays were carried out in black Nunc flatbottomed microtitre plates (Sigma-Aldrich; \#P8741), and technical triplicates were included for each sample. Once mixed, reaction plates were incubated for $30 \mathrm{~min}\left(37^{\circ} \mathrm{C}\right.$, static) before the addition of stop solution. Fluorescence was measured using a FLUOstar Omega plate reader (BMG LabTech), set to excitation and emission wavelengths of 360 and $450 \mathrm{~nm}$, respectively. A $1 \%$ gain was applied to the fluorescence measured by the reader. Blank fluorescence was subtracted from each sample reading prior to analysis.

\section{Statistics, data visualization and figure generation}

Figures were produced using R v3.5.1 [66], ggpubr v0.2.3 (https://github.com/kassambara/ggpubr), ggplot2 v3.2.1 [67], ggforce v0.3.1.9000 (https://github.com/thomasp85/ ggforce), and the Phandango web server [68]. Statistical tests were performed using $\mathrm{R}$ v3.5.1 [66]. Where required, figures were modified manually using InkScape v0.92.4 and Adobe Illustrator CC v23.1.1.

\section{RESULTS}

\section{Distribution of chitinase genes amongst $\boldsymbol{V}$. cholerae}

The key components of $V$. cholerae chitin catabolism summarized in Fig. 1 have been previously described [24, 45, 69]. The presence and absence of orthologues of each of the principal chitin-binding proteins and extracellular chitinases [45] known to be encoded by the V. cholerae 7PET reference strain N16961 (based on their N16961 locus identifiers) were identified in a pangenome calculated from $195 \mathrm{~V}$. cholerae genomes, plus three Vibrio species genomes used as an outgroup (Table S1). Genes that were annotated as encoding putative chitinases, as well as those genes known to be present in N16961, were identified in the pangenome (Tables S2 and S3). A V. cholerae phylogenetic tree was calculated using an SNV-only alignment of 2520 core genes taken from the pangenome, and the distribution of these chitinase genes across the phylogeny is presented in Fig. 2.

\section{chiA-2 is ubiquitous amongst diverse V. cholerae, but $g b p A$ and $c h i A-1$ are not}

The first, and most striking, observation made from these data was that $g b p A$ (VC_A0811) did not appear to be ubiquitous amongst all of the $V$. cholerae genomes included in this study. This was surprising because this gene had previously been reported to be ubiquitous amongst $V$. cholerae [70]. We found that $g b p A$ was present in only 189 of $195 \mathrm{~V}$. cholerae genomes (96.9\%; Fig. 2, Table S2). We manually inspected the genome assembly for each isolate which lacked $g b p A$, to guard against this being an artefact of the computational approach taken (Fig. S1).

Three genomic arrangements were observed at this locus - the presence of an intact VC_A0811 locus as found in $g b p A^{+}$genomes, a deletion of $g b p A$ and two adjacent genes (VC_A0811-VC_A0813), and the replacement of these three genes with additional sequence in the genome of NCTC 30 (Fig. S1). In order to ensure that the VC_A0811-VC_A0813 genes were not present at a different position in the NCTC 30 genome, we mapped the Illumina short-reads for this 


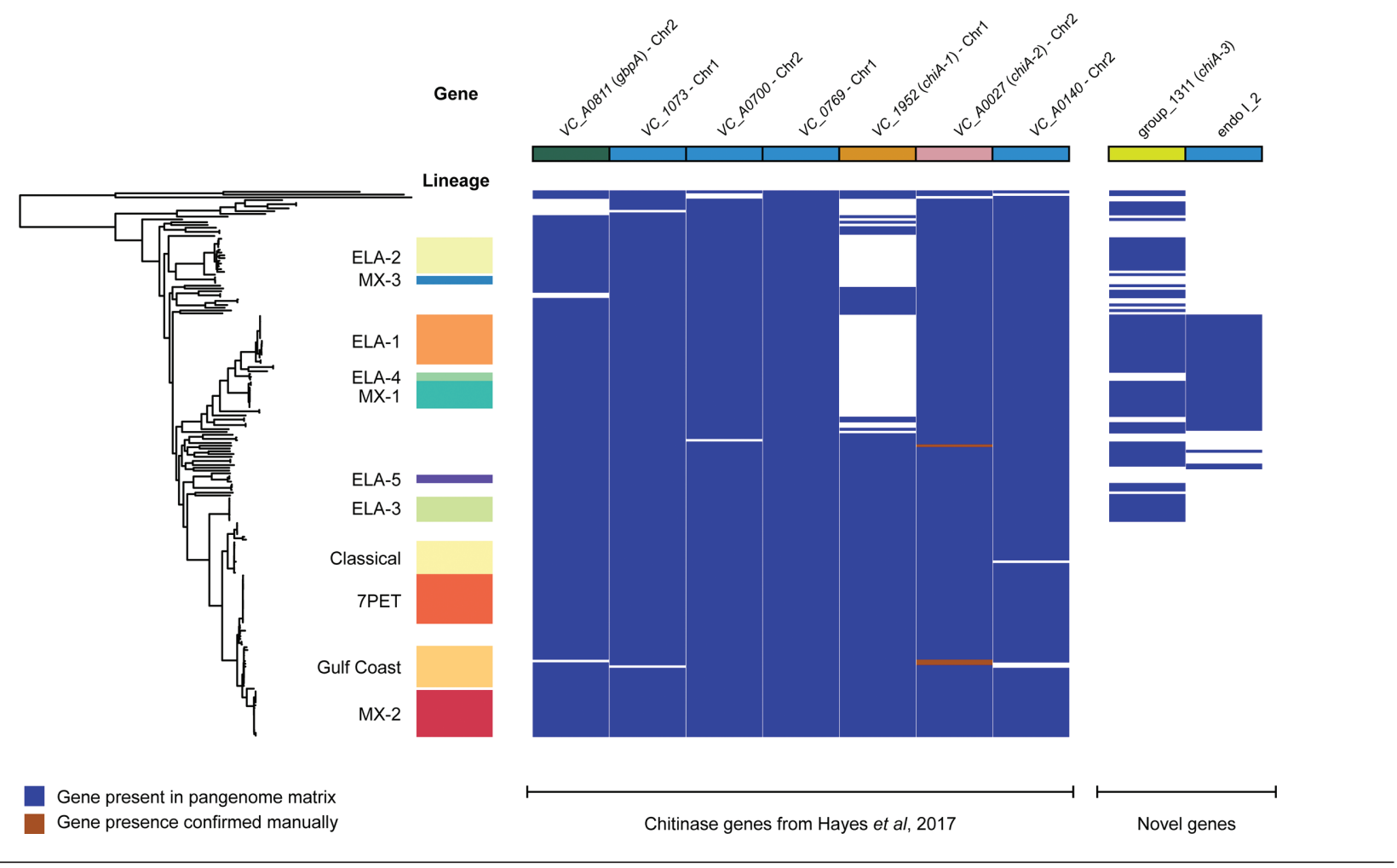

Fig. 2. Distribution of chitinase genes amongst diverse $V$. cholerae. Visualization of the presence and absence of genes encoding key $V$. cholerae chitinase enzymes and chitin adhesion factors (Fig. 1). The seven genes encoding putative V. cholerae endochitinases described by Hayes et al. [45] are listed, as well as two additional putative chitinases identified in this analysis. The figure was generated using Phandango [68]. Isolate assignments to $V$. cholerae lineages were taken verbatim from the literature [65, 89], and are named after Domman et al. [8]. Chromosomal location assigned to genes present in N16961. The presence of VC_A0027 in three genomes was confirmed manually, as described in the text. Colour coding of gbpA, chiA-1, chiA-2 and chiA-3 is consistent among figures in this paper.

isolate to the N16961 reference sequence and inspected the mapping coverage across this region. This confirmed that the absence of the genes VC_A0811-VC_A0813 from NCTC 30 was not a result of a mis-assembly (Fig. S2). The two genes adjacent to $g b p A, V C \_A 0812$ and $V C \_A 0813$, encode LapX and Lap, respectively. Both genes are putatively regulated by the HapR master quorum-sensing regulator, and encode proteins that were detected in an hapA mutant [71]. Both Lap and LapX were found to be putative components of the Type 2 secretome in N16961 [72], and lap has been used as a polymorphic locus in multilocus enzyme electrophoresis (MLEE) schemes for classifying $V$. cholerae $[73,74]$. We were unable to find published evidence linking these genes to GbpA activity or chitin adhesion more generally, although we note that lap and lapX are oriented in the same direction as $g b p A$, and we cannot exclude the possibility that these three genes are co-regulated or co-transcribed.

We found that VC_A0027 (encoding ChiA-2) was nearubiquitous, initially being detected in 192/195 V. cholerae (Fig. 2; Table S2). Manual inspection of the assemblies for those three isolates which appeared to lack the gene confirmed that the majority of this gene was in fact present; assembly and resultant annotation errors were likely to be responsible for this result (data not shown). This suggests that VC_A0027 is core to $V$. cholerae (195/195 genomes). This is consistent with ChiA-2 being the most highly expressed chitinase enzyme in the species, and with the observation that deletion of this gene alone causes a significant growth defect on minimal media containing chitin as a sole carbon source [45].

However, although VC_1952 (ChiA-1) was present in all pandemic isolates (defined as those isolates which were members of the 7PET and Classical lineages), it was not ubiquitous across the species, and was only identified in $61.2 \%$ of the non-pandemic $V$. cholerae in this dataset (101/165; Fig. 2; Table S2). This observation was surprising, because both ChiA-1 and ChiA-2 have been shown to be necessary for $V$. cholerae N16961 to grow in media supplemented with colloidal chitin [24]. Keymer and colleagues previously observed, using microarray approaches, that some diverse environmental isolates of $V$. cholerae varied in terms of their VC_1952 genotype [75]. We propose that our data recapitulate this observation, albeit in silico. We manually examined the region surrounding the VC_1952 locus in a subset of the genome assemblies for isolates lacking this gene, and found both that the gene was absent in its entirety, and that this did not appear to affect the genes adjacent to chiA-1 (Figs S3 and S4). Moreover, the distribution of putative chitinases (Fig. 2) suggested that isolates lacking ChiA-1 may encode additional 
a

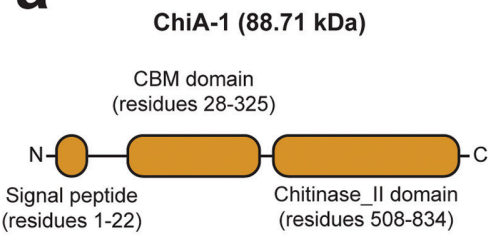

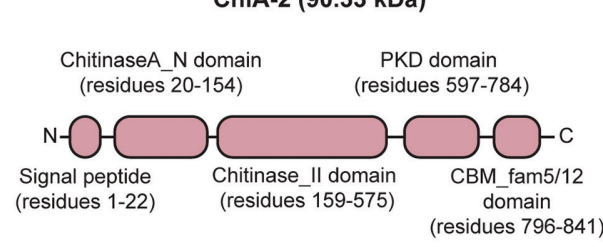

ChiA-2 (90.33 kDa)

(residues 796-841)

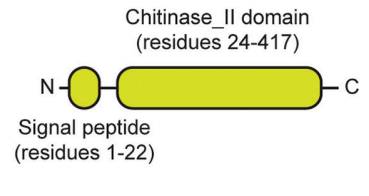

b

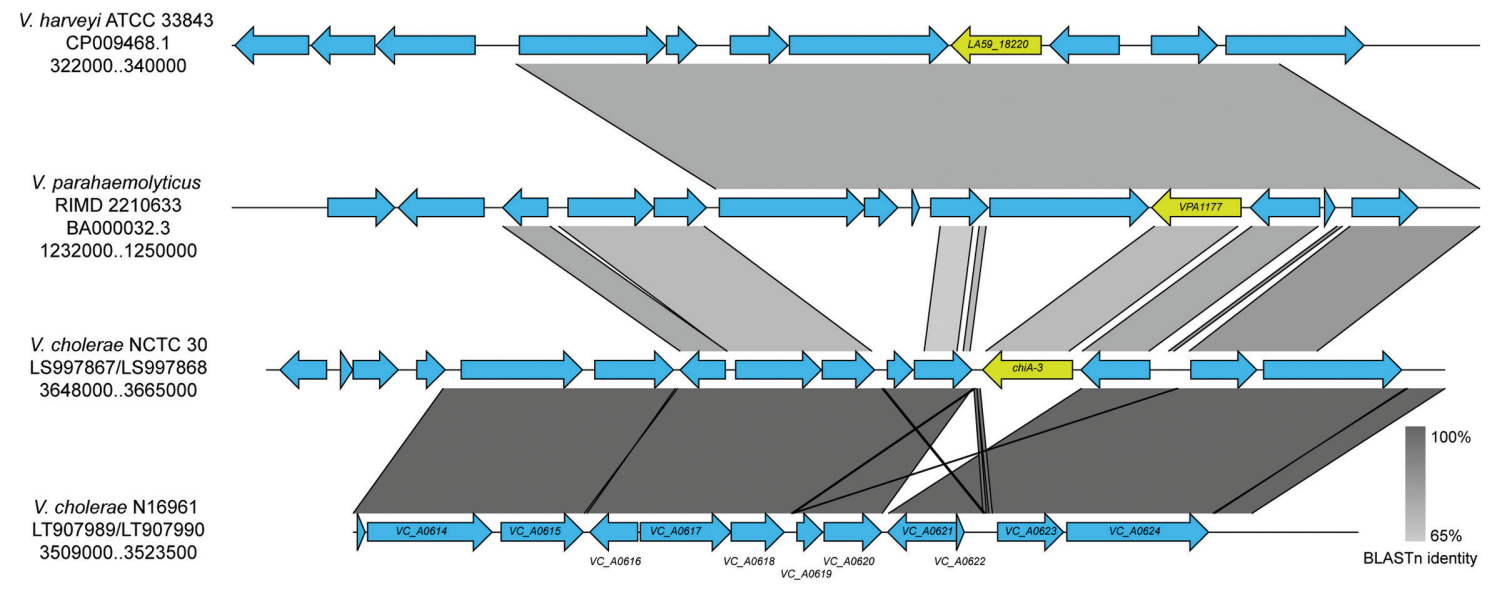

Fig. 3. ChiA-3 is a protein distinct from ChiA-1 and ChiA-2. (a) Cartoons of the protein domains predicted to be present in each of ChiA-1, ChiA-2 and ChiA-3 are presented. Predicted molecular weights are indicated. Proteins are not to scale. (b) chiA-3 is integrated between VC_A0620 and VC_A0621 in the smaller V. cholerae chromosome. This genomic position is conserved in Vibrio species which harbour chiA-3 orthologues [76, 79]. The figure was generated using Easyfig [61] and BLASTn comparisons [58].

chitinases. Since ChiA-1 is known to have a functional role in $V$. cholerae chitin metabolism, this led us to speculate that these additional putative chitinases, if functional, might be able to provide chitinase activity in the absence of ChiA-1.

\section{Identification and characterization of chiA-3}

Eleven gene clusters in the pangenome included genes with the annotation 'chitinase' or 'putative chitinase' (Table S3). Five of these were found only in one genome, of which four were found only in the non- $V$. cholerae outgroup. Of the remaining six genes, four are known to be present in N16961 (Tables S2 and S3). On further examination, the products of one of the two gene clusters, 'endo I_2', were not predicted in silico to contain a chitinase domain, although a putative chitin-binding domain was identified (Fig. S5; Table S3). The second gene identified was predicted to encode a protein containing a chitinase domain (Fig. 3a). The molecular weight $(47.69 \mathrm{kDa})$ and domain composition of the protein were distinct from those of chiA-2 and chiA-1 (Fig. 3a), as was the genomic context and location of the gene, which was inserted between VC_A0620 and VC_A0621 on chromosome 2 (Fig. 3b). This gene was therefore referred to as chiA-3, to differentiate it from the two previously described genes. chiA-3 was identified in 87 genomes, and was absent from all of the genomes belonging to both pandemic $V$. cholerae lineages included in this study. Additionally, 57 of the 67 isolates which lacked chiA-1 harboured chiA-3 (85.0\%).

In order to determine whether chiA-3 had been identified previously in other Vibrio species, the gene was compared to the nine genes listed by Hunt et al. as chitinases found in non-cholera vibrios [20] (Table 2). The most similar protein to ChiA-3 (76.57\% aa identity) was that encoded by VPA1177 (chiA, accession no. BAC62520.1), found in V. parahaemolyticus strain RIMD 2210633 (Table 2) [76]. VPA1177 encodes a 430 aa protein $(47.98 \mathrm{kDa})$ which previous genetic analyses have shown to make a minimal contribution to the ability of $V$. parahaemolyticus to degrade chitin; ChiA-2 (encoded by VPA0055, accession no. BAC61398.1) is the major protein responsible for this phenotype in $V$. parahaemolyticus [77]. Transcription of VPA 1177 has been shown to be significantly reduced in the presence of chitin [77]; however, the VPA1177 protein has been shown to be expressed by $V$. parahaemolyticus, albeit at very low levels in culture supernatants [36].

A previous report had also identified a functional secreted chitinase from Vibrio harveyi of a similar molecular weight $(47 \mathrm{kDa})$ to both VPA1177 and ChiA-3 [78]. The V. harveyi ATCC 33843 genome [79] contains a gene encoding a putative chitinase (BLASTP: $100 \%$ query coverage, $77.73 \%$ amino acid identity to ChiA-3; predicted molecular weight $48.0 \mathrm{kDa}$ ) in 
a

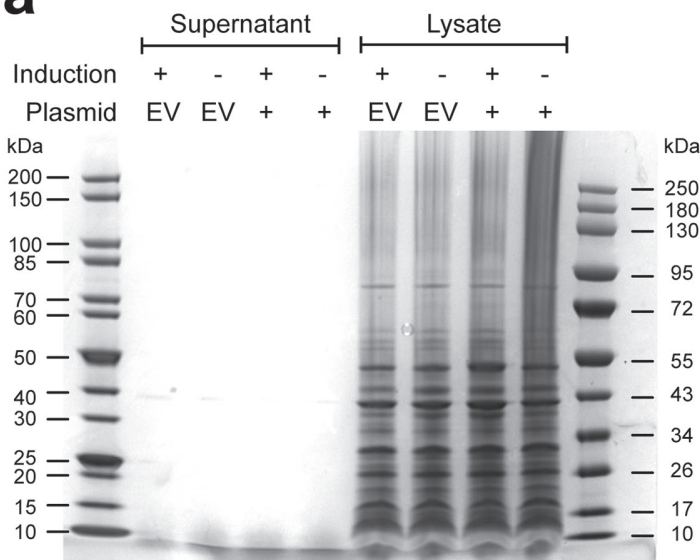

b

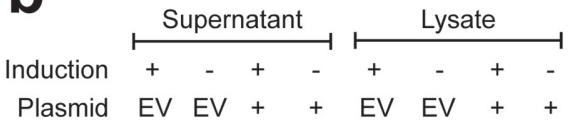

Fig. 4. Molecular cloning of chiA-3 and expression of ChiA-3-6xHis. (a) InstantBlue-stained acrylamide gel of proteins present in supernatants and cell pellet lysates from cultures grown at $23^{\circ} \mathrm{C}$ supplemented with arabinose (induction +) or glucose (induction -). No induced bands were easily discerned. (b) Western immunoblot produced from an identically loaded acrylamide gel to that presented in (a) run in parallel with the gel in (a) and probed with an $\alpha$-6xHis antibody (see Methods). A band corresponding to the expected molecular weight of ChiA-3-6xHis (48.51 kDa) was only detected in the cell pellet lysate of E. coli harbouring pMJD157 (plasmid +). This size is consistent with the retention of the fusion protein without the cleavage of the putative signal sequence. Protein ladders: NEB \#P7719S and \#P7717S. EV=empty vector (pBAD33).

a similar genomic context on chromosome 2 to that of chiA-3 in NCTC 30 (Fig. 3b). This is distinct from the location of the functionally characterized chiA gene (LA59_20935) which encodes an 850 aa ChiA chitinase precursor (accession no. Q9AMP1 [37, 80, 81]), and from other functionally characterized $V$. harveyi $\beta$ - $N$-acetylglucosaminidases [38]. This $V$. harveyi protein is also $90.9 \%$ identical to VPA1177. As well as their high amino acid identity, each of these proteins was predicted to contain similar domain compositions and configurations across the three species (Fig. S6). It is reasonable to infer that these enzymes are orthologues of ChiA-3.

Since VPA1177 has been shown to be transcribed [77] and to produce a translated protein in $V$. parahaemolyticus [36], we sought to determine whether the product of chiA-3 from $V$. cholerae had chitinase activity. We amplified the gene from the genome of NCTC 30, a non-pandemic lineage V. cholerae, and cloned it directionally into $\mathrm{pBAD} 33$ such that expression of the gene was governed by the arabinose-inducible $P_{B A D}$ promoter and the translated product linked to a C-terminal 6xHis tag, similar to previous reports [40, 45] (denoted pMJD157, Fig. S7).

E. coli harbouring pMJD157 produced a His-tagged protein of the expected molecular weight that was retained in the cell pellet when cultured with arabinose at $23^{\circ} \mathrm{C}$ (Fig. 4). We used a commercial fluorogenic assay for chitinase activity which relies on the hydrolysis of 4-methylumbelliferyl (4-MU) chitin analogues to detect chitinase activity. A similar assay has been used previously to assay chitinase activity in vibrios [78]. We found that samples from E. coli cultures expressing 6xHis-tagged ChiA-3 demonstrated statistically significant activity on 4-MU-linked substrates (Fig. 5). These data were consistent with the His-tagged protein detected in Fig. 4)
(ChiA-3-6xHis) having endochitinase and chitobiosidase activities, but lacking $\beta$ - $N$-acetylglucosaminidase activity. These results are also fully consistent with previous studies of $V$. cholerae chitinase enzyme activity when expressed in E. coli [48].

\section{DISCUSSION}

In this study, we present three major observations: first, that $g b p A$ is not ubiquitous amongst $V$. cholerae; second, we show that there is additional variability in the chitinase genes harboured by diverse $V$. cholerae, which show phylogenetic signals in their distribution; third, we present functional evidence that one of these putatively novel genes encodes a protein with chitinase activity.

The fact that $g b p A$ is not present in all $V$. cholerae is important, given that $g b p A$ had previously been suggested to be a candidate diagnostic gene for the detection of $V$. cholerae $[70,82]$. This was based both on the high level of conservation of $g b p A$ amongst tested $V$. cholerae, and on the number of differences between $g b p A$ in V. cholerae and alleles found in other Vibrio species [70]. In addition to our results, others have noted that $g b p A$ can be found in non-cholera vibrios and in nonpathogenic $V$. cholerae, suggesting that this makes $g b p A$ an unreliable marker for quantitative study of $V$. cholerae [83].

The biological consequences of the absence of $g b p A$ from these bacteria is interesting to consider. As discussed previously, GbpA is an important factor in both environmental and pathogenic colonization. The fact that $g b p A$ is absent from non-pandemic $V$. cholerae that appear to be basal to the rest of the species (Fig. 2) suggests that its role in pathogenicity may be more complex than previously thought. It might be 

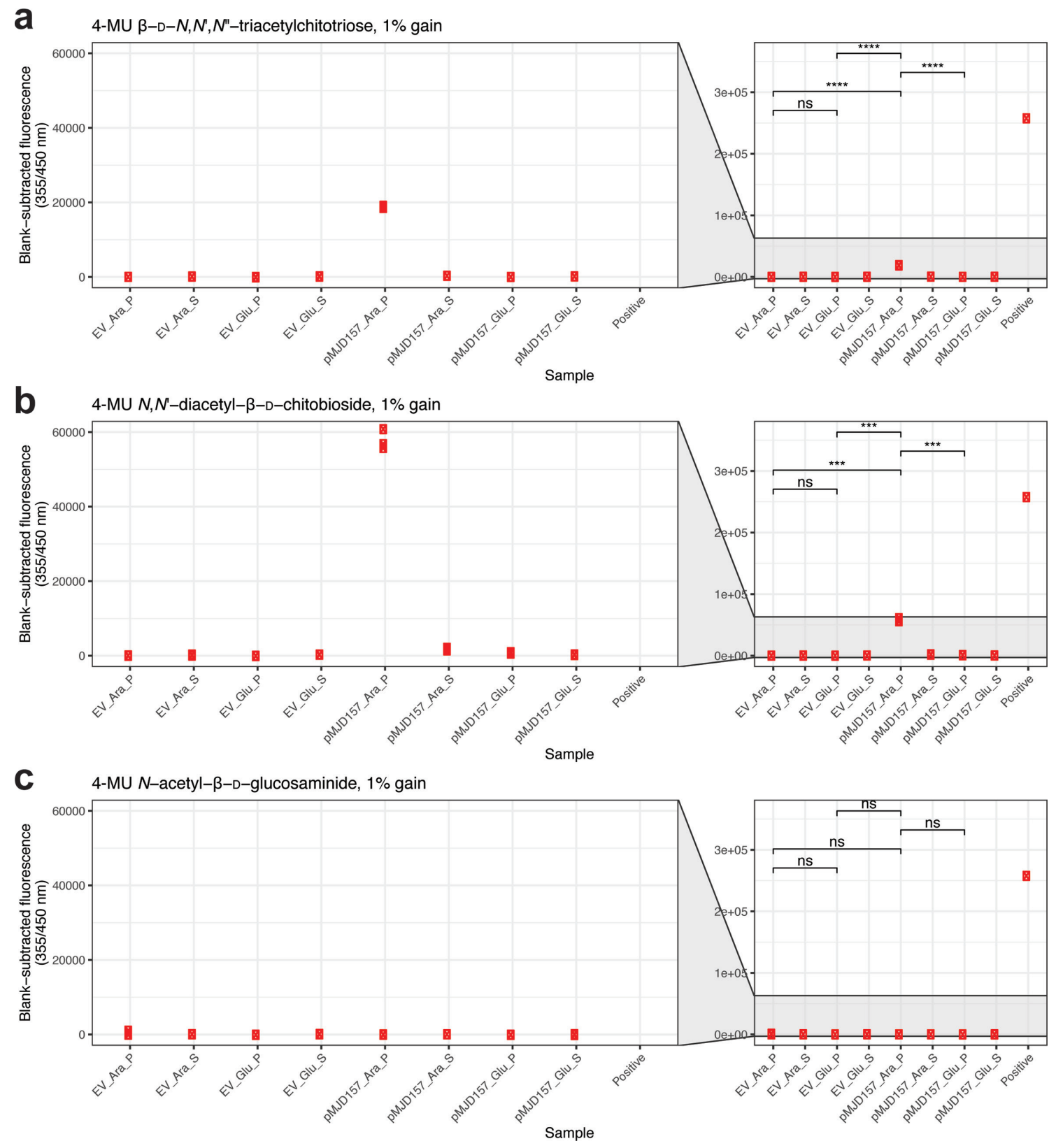

Fig. 5. ChiA-3-6xHis displays chitobiosidase and endochitinase activities, but not $\beta$ - $N$-acetylglucosaminidase activity. Lysates and supernatants included in Fig. 4(a, b) were assayed for chitinase enzyme activity using a fluorometric chitinase assay kit (see Methods for details). Lysed cells from Escherichia coli cultures harbouring pMJD157 and cultured in the presence of arabinose were the only samples which produced detectable and statistically significant signals on triacetylchitotriose and chitobiose substrates (a, b). No signal was detected in the presence of glucosaminide substrate (c). All plots are scaled equivalently. $P=$ pellet; $S=$ supernatant; $E V=e m p t y$ vector (pBAD33). Parametric $t$-tests were performed where indicated, testing for statistically significant differences between cell pellets of both induced and non-induced cultures harbouring each plasmid (effect of arabinose induction on chitinase activity), as well as between pellets from induced cultures (effect of plasmid insert on activity). ns=not significant; ${ }^{* * *} P<0.001 ;{ }^{* * * *} P<0.0001$.

that acquisition of $g b p A$ by $V$. cholerae was an important step in its evolution as a human pathogen. Conversely, because several of the isolates in the lineage lacking $g b p A$ are of clinical as well as environmental origin $[8,65,84-86]$, including some which were isolated from cases of acute or 'choleraic' diarrhoea $[65,84,85]$, it might be that $g b p A$ may not be essential for pathogenic colonization. It remains to be seen whether the natural absence of $g b p A$ affects the ability of such $V$. cholerae to colonize both the intestinal mucosa and chitinous surfaces. The roles played by other adhesins in these diverse $V$. cholerae, such as MSHA, should also be considered in the future. 
Although ChiA-3 orthologues have been examined in other vibrios, we believe that this is the first report of this gene in $V$. cholerae, and the first report that the V. cholerae chiA-3 gene encodes a functional chitinase. The fact that $\operatorname{chiA}-3$ was found only in non-pandemic V. cholerae is also intriguing. It is not yet known whether non-pandemic $V$. cholerae harbouring chiA-3 can respire chitin as effectively, or more effectively, than N16961 or other laboratory strains. A limitation of our study is that the genome sequences available to us are derived from isolates that are biased towards clinical cases of disease. Thus, our study may underestimate the diversity within the species [87], as well as the numbers of chitinases present in the species. Future research should include sequencing the genomes of both clinical and non-clinical $V$. cholerae, to address this implicit bias in genomic datasets.

There are fundamental differences between $V$. cholerae from pandemic and non-pandemic lineages, in terms of both their ability to cause cholera epidemics and their basic biology. We still do not fully understand these differences, but in order to do so, we must study $V$. cholerae pathogenicity in conjunction with more fundamental biological processes. It is currently unclear whether variation in the complements of chitinases and chitin-binding proteins encoded by $V$. cholerae have physiological consequences for different lineages of the species. However, given the importance of these genes to pathogenicity [30,40], environmental lifestyles [24] and natural competence [29], it is plausible that these differences reflect differences in the ecological niches occupied by different lineages of the species. Research in this area may provide further insights into the genetic and biochemical differences between $V$. cholerae lineages that cause dramatically different patterns of disease worldwide. Collectively, these findings underline the fact that, as we continue to study diverse $V$. cholerae, our understanding of the nuance and specifics of this species will improve and be refined.

Funding information

T.G.F. was supported by an Amgen Foundation Scholarship to the University of Cambridge. M.J.D. is a Junior Research Fellow at Churchill College, Cambridge, and was supported previously by a Wellcome Sanger Institute PhD Studentship. G.A.B. is an EBI-Sanger Postdoctoral (ESPOD) Fellow. This research was funded in whole, or in part, by the Wellcome Trust (grant 206194). For the purpose of Open Access, the authors have applied a CC BY public copyright licence to any Author Accepted Manuscript version arising from this submission.

\section{Acknowledgements}

We thank Rita Monson for helpful discussions and comments throughout the project, and members of the Thomson group for feedback on the manuscript. We thank Sally Kay for logistical support, Charlotte Tolley for the gift of reagents, and the Wellcome Sanger Institute (WSI) Pathogen Informatics team for help with data management. We also thank the WSI Parasites and Microbes Administration team, particularly Kate Auger and Joseph Woolfolk, for operational and administrative assistance in the course of this work.

\section{Author contributions}

N.R.T. supervised the work. T.G.F. performed genomic analysis with assistance from M.J.D. and G.A.B. M.J.D. carried out experimental work. T.G.F. and M.J.D. wrote the manuscript, with major contributions from N.R.T. All authors interpreted the results, contributed to the editing of the manuscript, and read and approved the final version of the manuscript.

\section{Conflicts of interest}

The authors declare that there are no conflicts of interest.

\section{References}

1. Morris JG, Black RE. Cholera and other vibrioses in the United States. N Engl J Med 1985:312:343-350.

2. Pollitzer R, Swaroop S, Burrows W. Cholera. World Health Organization, 1959

3. Kaper JB, Morris JG, Levine MM. Cholera. Clin Microbiol Rev 1995;8:48-86

4. Devault AM, Golding GB, Waglechner N, Enk JM, Kuch M, et al. Second-pandemic strain of Vibrio cholerae from the Philadelphia cholera outbreak of 1849. N Engl J Med 2014:370:334-340.

5. Cvjetanovic B, Barua D. The seventh pandemic of cholera. Nature 1972;239:137-138.

6. Furniss AL, Lee JV, Donovan TJ. The Vibrios. London: His Majesty's Stationery Office (H.M.S.O); 1978.

7. Weill F-. X, Domman D, Njamkepo E, Tarr C, Rauzier J, et al. Genomic history of the seventh pandemic of cholera in Africa. Science 2017;358:785-789.

8. Domman D, Quilici ML, Dorman MJ, Njamkepo E, Mutreja A, et al. Integrated view of Vibrio cholerae in the Americas. Science 2017:358:789-793.

9. Oprea M, Njamkepo E, Cristea D, Zhukova A, Clark CG, et al. The seventh pandemic of cholera in Europe revisited by microbial genomics. Nat Commun 2020;11:5347.

10. Mutreja A, Kim DW, Thomson NR, Connor TR, Lee JH, et al. Evidence for several waves of global transmission in the seventh cholera pandemic. Nature 2011;477:462-465

11. Karlsson SL, Thomson N, Mutreja A, Connor T, Sur D, et al. Retrospective analysis of serotype switching of Vibrio cholerae 01 in a cholera endemic region shows it is a non-random process. PLOS Negl Trop Dis 2016;10:e0005044.

12. Chun J, Grim CJ, Hasan NA, Lee JH, Choi SY, et al. Comparative genomics reveals mechanism for short-term and long-term clonal transitions in pandemic Vibrio cholerae. Proc Natl Acad Sci U S A 2009:106:15442-15447.

13. Clemens JD, Nair GB, Ahmed T, Qadri F, Holmgren J. Cholera. Lancet 2017;390:1539-1549.

14. Jones MK, Oliver JD. Vibrio vulnificus: Disease and pathogenesis. Infect Immun 2009;77:1723-1733.

15. Daniels NA, MacKinnon L, Bishop R, Altekruse S, Ray B, et al. Vibrio parahaemolyticus infections in the United States, 1973-1998. J Infect Dis 2000;181:1661-1666.

16. Goarant C, Reynaud Y, Ansquer D, de Decker S, Saulnier D, et al. Molecular epidemiology of Vibrio nigripulchritudo, a pathogen of cultured penaeid shrimp (Litopenaeus stylirostris) in New Caledonia. Syst Appl Microbiol 2006;29:570-580.

17. Goarant C, Ansquer D, Herlin J, Domalain D, Imbert F, et al. "Summer Syndrome" in Litopenaeus stylirostris in New Caledonia: Pathology and epidemiology of the etiological agent, Vibrio nigripulchritudo. Aquaculture 2006;253:105-113.

18. Frans I, Michiels CW, Bossier P, Willems KA, Lievens B, et al. Vibrio anguillarum as a fish pathogen: Virulence factors, diagnosis and prevention. J Fish Dis 2011;34:643-661.

19. Zhang X-. H, He X, Austin B. Vibrio harveyi: A serious pathogen of fish and invertebrates in mariculture. Mar Life Sci Technol 2020;2:231-245.

20. Hunt DE, Gevers D, Vahora NM, Polz MF. Conservation of the chitin utilization pathway in the Vibrionaceae. Appl Environ Microbiol 2008;74:44-51.

21. Keyhani NO, Roseman S. Physiological aspects of chitin catabolism in marine bacteria. Biochim Biophys Acta - Gen Subj 1999;1473:108-122. 
22. Froelich B, Oliver J. Increases in the amounts of Vibrio spp. in oysters upon addition of exogenous bacteria. Appl Environ Microbiol 2013;79:5208-5213.

23. Kaneko T, Colwell RR. Adsorption of Vibrio parahaemolyticus onto chitin and copepods. Appl Microbiol 1975;29:269-274.

24. Meibom KL, Li XB, Nielsen AT, Wu C-Y, Roseman S, et al. The Vibrio cholerae chitin utilization program. Proc Natl Acad Sci U S A 2004:101:2524-2529

25. Nalin DR, Daya V, Reid A, Levine MM, Cisneros L. Adsorption and growth of Vibrio cholerae on chitin. Infect Immun 1979;25:768-770.

26. Huq A, Small EB, West PA, Huq MI, Rahman R, et al. Ecological relationships between Vibrio cholerae and planktonic crustacean copepods. Appl Environ Microbiol 1983;45:275-283.

27. Lo Scrudato $M$, Blokesch M. A transcriptional regulator linking quorum sensing and chitin induction to render Vibrio cholerae naturally transformable. Nucleic Acids Res 2013;41:3644-3658.

28. Antonova ES, Hammer BK. Genetics of natural competence in Vibrio cholerae and other Vibrios. Microbiol Spectr 2015;3.

29. Meibom KL, Blokesch M, Dolganov NA, Wu C-Y, Schoolnik GK. Chitin induces natural competence in Vibrio cholerae. Science 2005:310:1824-1827

30. Mondal M, Nag D, Koley H, Saha DR, Chatterjee NS. The Vibrio cholerae extracellular chitinase ChiA2 is important for survival and pathogenesis in the host intestine. PLOS ONE 2014;9:e103119.

31. Conner JG, Teschler JK, Jones CJ, Yildiz FH. Staying alive: Vibrio cholerae's cycle of environmental survival, transmission, and dissemination. Microbiol Spectr 2016;4

32. Keyhani NO, Roseman S. The chitin catabolic cascade in the marine bacterium Vibrio furnissii: Molecular cloning, isolation, and characterization of a periplasmic $\beta$ - $N$-acetylglucosaminidase. J Biol Chem 1996:271:33425-33432

33. Keyhani NO, Roseman S. The chitin catabolic cascade in the marine bacterium Vibrio furnissii: Molecular cloning, isolation, and characterization of a periplasmic chitodextrinase. J Biol Chem 1996:271:33414-33424

34. Chitlaru E, Roseman S. Molecular cloning and characterization of a novel $\beta$ - $N$-acetyl-D-glucosaminidase from Vibrio furnissii. J Biol Chem 1996;271:33433-33439.

35. Jung B-. 0, Roseman S, Park JK. The central concept for chitin catabolic cascade in marine bacterium, Vibrios. Macromol Res 2008:16:1-5

36. Hirano T, Okubo M, Tsuda H, Yokoyama M, Hakamata W, et al. Chitin heterodisaccharide, released from chitin by chitinase and chitin oligosaccharide deacetylase, enhances the chitin-metabolizing ability of Vibrio parahaemolyticus. J Bacteriol 2019;201:e00270-19.

37. Suginta W, Vongsuwan A, Songsiriritthigul C, Prinz H, Estibeiro $P$, et al. An endochitinase A from Vibrio carchariae: Cloning, expression, mass and sequence analyses, and chitin hydrolysis. Arch Biochem Biophys 2004:424:171-180.

38. Suginta W, Chuenark D, Mizuhara M, Fukamizo T. Novel $\beta-N-$ acetylglucosaminidases from Vibrio harveyi 650: Cloning, expression, enzymatic properties, and subsite identification. BMC Biochem 2010:11:40

39. Wortman AT, Somerville CC, Colwell RR. Chitinase determinants of Vibrio vulnificus: Gene cloning and applications of a chitinase probe. Appl Environ Microbiol 1986;52:142-145.

40. Kirn TJ, Jude BA, Taylor RK. A colonization factor links Vibrio cholerae environmental survival and human infection. Nature 2005:438:863-866.

41. Bhowmick R, Ghosal A, Das B, Koley H, Saha DR, et al. Intestinal adherence of Vibrio cholerae involves a coordinated interaction between colonization factor GbpA and mucin. Infect Immun 2008:76:4968-4977.

42. Wong E, Vaaje-Kolstad G, Ghosh A, Hurtado-Guerrero R, Konarev PV, et al. The Vibrio cholerae colonization factor GbpA possesses a modular structure that governs binding to different host surfaces. PLoS Pathog 2012;8:e1002373.
43. Loose JSM, Forsberg Z, Fraaije MW, Eijsink VGH, Vaaje-Kolstad G A rapid quantitative activity assay shows that the Vibrio cholerae colonization factor GbpA is an active lytic polysaccharide monooxygenase. FEBS Lett 2014;588:3435-3440.

44. Jude BA, Martinez RM, Skorupski K, Taylor RK. Levels of the secreted Vibrio cholerae attachment factor GbpA are modulated by quorum-sensing-induced proteolysis. I Bacteriol 2009:191:6911-6917.

45. Hayes CA, Dalia TN, Dalia AB. Systematic genetic dissection of chitin degradation and uptake in Vibrio cholerae. Environ Microbiol 2017:19:4154-4163.

46. Li X, Roseman S. The chitinolytic cascade in Vibrios is regulated by chitin oligosaccharides and a two-component chitin catabolic sensor/kinase. Proc Natl Acad Sci USA 2004;101:627-631.

47. Dalia $A B$. RpoS is required for natural transformation of Vibrio cholerae through regulation of chitinases. Environ Microbiol 2016;18:3758-3767.

48. Connell TD, Metzger DJ, Lynch J, Folster JP. Endochitinase is transported to the extracellular milieu by the eps-encoded general secretory pathway of Vibrio cholerae. I Bacteriol 1998:180:5591-5600

49. Soysa HSM, Aunkham A, Schulte A, Suginta W. Single-channel properties, sugar specificity, and role of chitoporin in adaptive survival of Vibrio cholerae type strain 01. J Biol Chem 2020;295:9421-9432.

50. Bankevich A, Nurk S, Antipov D, Gurevich AA, Dvorkin M, et al. SPAdes: A new genome assembly algorithm and its applications to single-cell sequencing. J Comput Biol 2012;19:455-477.

51. Page AJ, De Silva N, Hunt M, Quail MA, Parkhill J, et al. Robust high-throughput prokaryote de novo assembly and improvement pipeline for Illumina data. Microb Genom 2016;2:e000083.

52. Seemann T. Prokka: Rapid prokaryotic genome annotation. Bioinformatics 2014:30:2068-2069.

53. O'Leary NA, Wright MW, Brister JR, Ciufo S, Haddad D, et al. Reference sequence (RefSeq) database at NCBI: Current status, taxonomic expansion, and functional annotation. Nucleic Acids Res 2016:44:D733-D745.

54. Page AJ, Cummins CA, Hunt M, Wong VK, Reuter S, et al. Roary: Rapid large-scale prokaryote pan genome analysis. Bioinformatics 2015;31:3691-3693.

55. Capella-Gutiérrez S, Silla-Martínez JM, Gabaldón T. trimAl: A tool for automated alignment trimming in large-scale phylogenetic analyses. Bioinformatics 2009;25:1972-1973.

56. Page AJ, Taylor B, Delaney AJ, Soares J, Seemann T, et al. SNPsites: Rapid efficient extraction of SNPs from multi-FASTA alignments. Microb Genom 2016;2:e000056.

57. Nguyen L-. T, Schmidt HA, von Haeseler A, Minh BQ. IQ-TREE: A fast and effective stochastic algorithm for estimating maximumlikelihood phylogenies. Mol Biol Evol 2015;32:268-274.

58. Altschul SF, Gish W, Miller W, Myers EW, Lipman DJ. Basic local alignment search tool. J Mol Biol 1990;215:403-410.

59. Jones $\mathrm{P}$, Binns $\mathrm{D}$, Chang $\mathrm{H}-\mathrm{Y}$, Fraser $\mathrm{M}$, Li W, et al. InterProScan 5: Genome-scale protein function classification. Bioinformatics 2014;30:1236-1240.

60. Carver TJ, Rutherford KM, Berriman M, Rajandream M-. A, Barrell BG, et al. ACT: The Artemis comparison tool. Bioinformatics 2005;21:3422-3423.

61. Sullivan MJ, Petty NK, Beatson SA. Easyfig: A genome comparison visualizer. Bioinformatics 2011;27:1009-1010.

62. Harris SR, Feil EJ, Holden MTG, Quail MA, Nickerson EK, et al. Evolution of MRSA during hospital transmission and intercontinental spread. Science 2010;327:469-474.

63. Carver T, Böhme U, Otto TD, Parkhill J, Berriman M. BamView: Viewing mapped read alignment data in the context of the reference sequence. Bioinformatics 2010;26:676-677.

64. Rutherford K, Parkhill J, Crook J, Horsnell T, Rice $P$, et al. Artemis: Sequence visualization and annotation. Bioinformatics 2000;16:944-945. 
65. Dorman MJ, Kane L, Domman D. Turnbull JD, Cormie C et al. The history, genome and biology of NCTC 30: A non-pandemic Vibrio cholerae isolate from World War One. Proc R Soc B: Biol Sci 2019;286:20182025

66. R Core Team. R: a Language and Environment for Statistical Computing. R Foundation for Statistical Computing, Vienna, Austria, 2018.

67. Wickham H. ggplot2: Elegant Graphics for Data Analysis. SpringerVerlag New York, 2016.

68. Hadfield J, Croucher NJ, Goater RJ, Abudahab K, Aanensen DM, et al. Phandango: An interactive viewer for bacterial population genomics. Bioinformatics 2018;34:292-293.

69. Reddi G, Pruss K, Cottingham KL, Taylor RK, Almagro-Moreno S. Catabolism of mucus components influences motility of Vibrio cholerae in the presence of environmental reservoirs. PLOS ONE 2018;13:e0201383.

70. Stauder M, Huq A, Pezzati E, Grim CJ, Ramoino P, et al. Role of GbpA protein, an important virulence-related colonization factor, for Vibrio cholerae's survival in the aquatic environment. Environ Microbiol Rep 2012;4:439-445.

71. Vaitkevicius K, Lindmark B, Ou G, Song T, Toma C, et al. A Vibrio cholerae protease needed for killing of Caenorhabditis elegans has a role in protection from natural predator grazing. Proc Natl Acad Sci U S A 2006;103:9280-9285.

72. Sikora AE, Zielke RA, Lawrence DA, Andrews PC, Sandkvist M. Proteomic analysis of the Vibrio cholerae type II secretome reveals new proteins, including three related serine proteases. J Biol Chem 2011:286:16555-16566.

73. Farfán $M$, Miñana $D$, Fusté MC, Lorén JG. Genetic relationships between clinical and environmental Vibrio cholerae isolates based on multilocus enzyme electrophoresis. Microbiology 2000;146:2613-2626

74. Farfán M, Miñana-Galbis D, Fusté MC, Lorén JG. Allelic diversity and population structure in Vibrio cholerae 0139 Bengal based on nucleotide sequence analysis. J Bacteriol 2002;184:1304-1313.

75. Keymer DP, Miller MC, Schoolnik GK, Boehm AB. Genomic and phenotypic diversity of coastal Vibrio cholerae strains is linked to environmental factors. Appl Environ Microbiol 2007;73:3705-3714.

76. Makino K, Oshima K, Kurokawa K, Yokoyama K, Uda T, et al. Genome sequence of Vibrio parahaemolyticus: a pathogenic mechanism distinct from that of $V$ cholerae. Lancet 2003;361:743-749.

77. Debnath A, Mizuno T, Miyoshi S. Regulation of chitin-dependent growth and natural competence in Vibrio parahaemolyticus. Microorganisms 2020;8:1303.
78. Svitil AL, Chadhain S, Moore JA, Kirchman DL. Chitin degradation proteins produced by the marine bacterium Vibrio harveyi growing on different forms of chitin. Appl Environ Microbiol 1997;63:408-413.

79. Wang Z, Hervey WJ, Kim S, Lin B, Vora GJ. Complete genome sequence of the bioluminescent marine bacterium Vibrio harveyi ATCC 33843 (392 [MAV]). Genome Announc 2015:3:e01493-14.

80. Suginta $W$, Sirimontree $P$, Sritho N, Ohnuma T, Fukamizo T. The chitin-binding domain of a GH-18 chitinase from Vibrio harveyi is crucial for chitin-chitinase interactions. Int J Biol Macromol 2016:93:1111-1117.

81. Songsiriritthigul C, Pantoom S, Aguda AH, Robinson RC, Suginta W. Crystal structures of Vibrio harveyi chitinase A complexed with chitooligosaccharides: Implications for the catalytic mechanism. J Struct Biol 2008;162:491-499.

82. Vezzulli L, Stauder M, Grande C, Pezzati E, Verheye HM, et al. gbpA as a novel qPCR target for the species-specific detection of Vibrio cholerae 01, 0139, non-01/non-0139 in environmental, stool, and historical continuous plankton recorder samples. PLOS ONE 2015;10:e0123983.

83. Nasreen T, Hussain NAS, Islam MT, Orata FD, Kirchberger PC, et al. Simultaneous quantification of Vibrio metoecus and Vibrio cholerae with its 01 serogroup and toxigenic subpopulations in environmental reservoirs. Pathogens 2020;9:1053.

84. Chowdhury F, Mather AE, Begum YA, Asaduzzaman M, Baby N, et al. Vibrio cholerae serogroup 0139: Isolation from cholera patients and asymptomatic household family members in Bangladesh between 2013 and 2014. PLoS Negl Trop Dis 2015:9:e0004183.

85. Gardner AD, Venkatraman KV. The antigens of the cholera group of Vibrios. J Hyg (Lond) 1935;35:262-282.

86. Hasan NA, Choi SY, Eppinger M, Clark PW, Chen A, et al. Genomic diversity of 2010 Haitian cholera outbreak strains. Proc Natl Acad Sci USA 2012;109:E2010-E2017.

87. Aydanian A, Tang L, Chen Y, Morris JG Jr, Olsen P, et al. Genetic relatedness of selected clinical and environmental non-01/0139 Vibrio cholerae. Int J Infect Dis 2015;37:152-158.

88. Guzman LM, Belin D, Carson MJ, Beckwith J. Tight regulation, modulation, and high-level expression by vectors containing the arabinose PBAD promoter. J Bacteriol 1995;177:4121-4130.

89. Dorman MJ, Kane L, Domman D, Turnbull JD, Cormie C, et al. Table S1 from The history, genome and biology of NCTC 30: A nonpandemic Vibrio cholerae isolate from World War One. Figshare 2019.

\section{Five reasons to publish your next article with a Microbiology Society journal}

1. The Microbiology Society is a not-for-profit organization.

2. We offer fast and rigorous peer review - average time to first decision is 4-6 weeks.

3. Our journals have a global readership with subscriptions held in research institutions around the world.

4. $80 \%$ of our authors rate our submission process as 'excellent' or 'very good'.

5. Your article will be published on an interactive journal platform with advanced metrics

Find out more and submit your article at microbiologyresearch.org. 